\title{
Romantic Name for a Deadly Condition: Kissing Aneurysms of the Pericallosal Artery - A Case Report
}

Przemyslaw M. Waszak, ${ }^{1}$ Agnieszka Paturej, ${ }^{1}$ Janusz Springer, ${ }^{1}$ Katarzyna Baranowska, ${ }^{1}$ Barbara Brzeska, ${ }^{1}$ Katarzyna Aleksandrowicz, ${ }^{1}$ Tomasz Szmuda, ${ }^{2}$ Hanna Garnier.

\begin{abstract}
Background: Kissing aneurysms are two independent but adjacent aneurysms protruding from two contralateral arterial locations. This report describes a successfully treated case of kissing aneurysms at the Department of Neurosurgery, Medical University of Gdansk. Case: A 45-year-old asymptomatic woman was diagnosed with unruptured bilateral aneurysms located in the pericallosal-callosomarginal division. Her medical history included a previous intracranial aneurysm and arterial hypertension. The patient underwent a successful treatment by surgical clipping and was discharged in good condition; neither disability nor neurologic deficit was noticed upon discharge. Surgical wound healing was complicated by an infection and resulted in a reoperation for the patient. Conclusion: The etiology of kissing aneurysms is still unknown and the best treatment method stills remains unclear. Thus, every case has to be carefully and individually assessed by an interdisciplinary team. As a result, patient transfer to an experienced neurosurgical center could be beneficial.
\end{abstract}

Keywords: Intracranial Aneurysm, Anterior Cerebral Artery, Microsurgery, Angiography, Kissing aneurysms (Source: MeSH, NLM).

About the Author: Przemyslaw M. Waszak is currently a 6th (final year) year medical student of the Medical University of Gdansk, Poland. He is also the founder and editor of the first Polish scientific handbook for medical students entitled "Idea - Research - Publication"
Submission: Dec 9, 2014

Acceptance: Apr 10, 2015

Publication: May 19, 2015

Process: Peer-reviewed

\section{Introduction}

The prevalence of intracranial aneurysm is estimated at approximately $3.2 \%$. $^{1}$ The major characteristics of aneurysms include type (saccular, fusiform, dissecting, mycotic, blood-blister-like, distal, etc.), size (micro, small, medium, large, giant etc.) and location (branching sites of anterior, medial or posterior cerebral artery etc.). Unruptured aneurysms are asymptomatic in most cases. When ruptured, they cause subarachnoid hemorrhage (SAH), also known as hemorrhagic stroke. Main SAH symptoms include sudden onset of severe headache, seizures, and neurologic deficits with rapid deterioration leading to loss of consciousness. Basic diagnosis involves computed tomography (CT) imaging or lumbar puncture. SAH represents a state of medical emergency; even when treated early, SAH is associated with mortality up to $50 \%$ (including neurologic deficits in many of the survivors). ${ }^{2}$ The main treatment options include open surgery with direct microsurgical clipping of the aneurysmal neck or an endovascular procedure that occludes the aneurysmal lumen. The risk of an aneurysm rupture depends on various risk factors such as the aneurysms' characteristics (localization, type and size) and the patients' characteristics and co-morbidities (hypertension, gender, cigarette smoking, alcohol intake, and prior history of aneurysm).3.4

Kissing aneurysms (KAs) are unusual locational phenomena of multiple aneurysms. Although the prevalence of multiple aneurysms can be up to $20 \%$ of all intracranial aneurysms, the KAs adjacent bilateral aneurysms arising from the same artery - are quite unique with an incidence as low as $0.2 \% .{ }^{5}$ Kissing aneurysms can be classified into two categories: type 1 represents aneurysmal necks that are located on the same parent artery

\section{Key Points:}

- The preferred treatment method of a single unruptured aneurysm re mains controversial, thus an unruptured KAs makes the decision process even more complicated.

- Surgery is difficult due to KAs dual arterial supply. The procedure should involve securing both aneurysms simultaneously, however this maneuver can lead to higher risk of intraoperative bleeding. - KAs still remain a challenge, because of their unknown etiology, difficult imaging and no officially-stated treatment method.

and type 2 exists where each aneurysmal neck is located on different parent arteries. ${ }^{6}$ The most common location for KAs is the internal carotid artery (ICA), but other sites such as the distal part of the anterior cerebral artery have also been reported.? However, KAs associated with the posterior arterial supply of the brain are mainly type 1. Kissing aneurysms are more common among women, especially middle-aged (40-59 years old). ${ }^{8}$ An interesting, but separate phenomenon is mirror-like (or twin) aneurysms that are located bilaterally on analogs of arteries (eg. left and right middle cerebral artery, MCA). ${ }^{9}$

Diagnosing KAs can be difficult in terms of determining the number or structure of aneurysms based on radiologic examination. It has been suggested that more than half of KAs are not been recognized preoperatively. ${ }^{6}$ Thus, the decision-making process can be particularly challenging. There is no recommended screening for intracranial aneurysms, so in most of the cases they are revealed only when they have ruptured. In a minority of the patients, the presence of an aneurysm is known beforehand. In these special cases, it is highly important to make a prompt diagnosis of unruptured aneurysms, evaluate the risk of bleeding, and consider its eventual prophylactic treatment.

'Medical University of Gdansk, Students' Scientific Association, Neurosurgery Department, Poland.

${ }^{2}$ Medical University of Gdansk, Neurosurgery Department, Poland.

Correspondence:

Przemyslaw M. Waszak

Address: Marii Skłodowskiej-Curie 3A, Gdańsk, Poland.

Email: p.waszak@gumed.edu.pl 
We present a case of unruptured KAs successfully treated surgically at the Neurosurgery Department of the Medical University of Gdansk. Informed consent was obtained from the patient for this study.

\section{The Case}

A 45-year-old asymptomatic woman was admitted to the Department of Neurosurgery for the planned surgical clipping of unruptured bilateral aneurysms located in the division of the anterior cerebral artery into the pericallosal and callosomarginal arteries (A2/3 kissing aneurysms, Figure 1 A, B). Kissing aneurysms were radiographically diagnosed, after the patient's previous aneurysm surgery.

The patient's medical history included hypertension, epilepsy, and 14 pack-years of smoking. According to the patient's family, in 2012 she had an extreme case of alcohol intoxication resulting in tonic seizures. Her surgical history included a previous surgical clipping of an unruptured aneurysm in 2012 performed on the division of the right middle cerebral artery (MCA) segments $M_{1} / M_{2}$. A computed tomography angiography (CTA) scan was performed and revealed bilateral (kissing) aneurysms $(4 \times 4.5 \mathrm{~mm}$ in size) occurring at the bifurcation of the anterior cerebral artery into the pericallosal and callosomarginal arteries (A2/3 division). This aneurysm was classified as type 1 as each aneurysmal neck was located on the same parent artery (Figure 1).

The patient's neurological status upon admission was normal and her general physical examination was unremarkable. Cerebral angiography was performed and the diagnosis of KAs was confirmed (Figure 1 C, D).

The patient underwent surgical clipping via a right frontal craniotomy approach under general anesthesia. The skull was opened with a free bone flap. Opening of the interhemispheric fissure was performed and the aneurysms were reached from the right and left side (Figure 2). Clips on the necks of the

Figure 1. Radiologic Images of the Patient's Aneurysms.

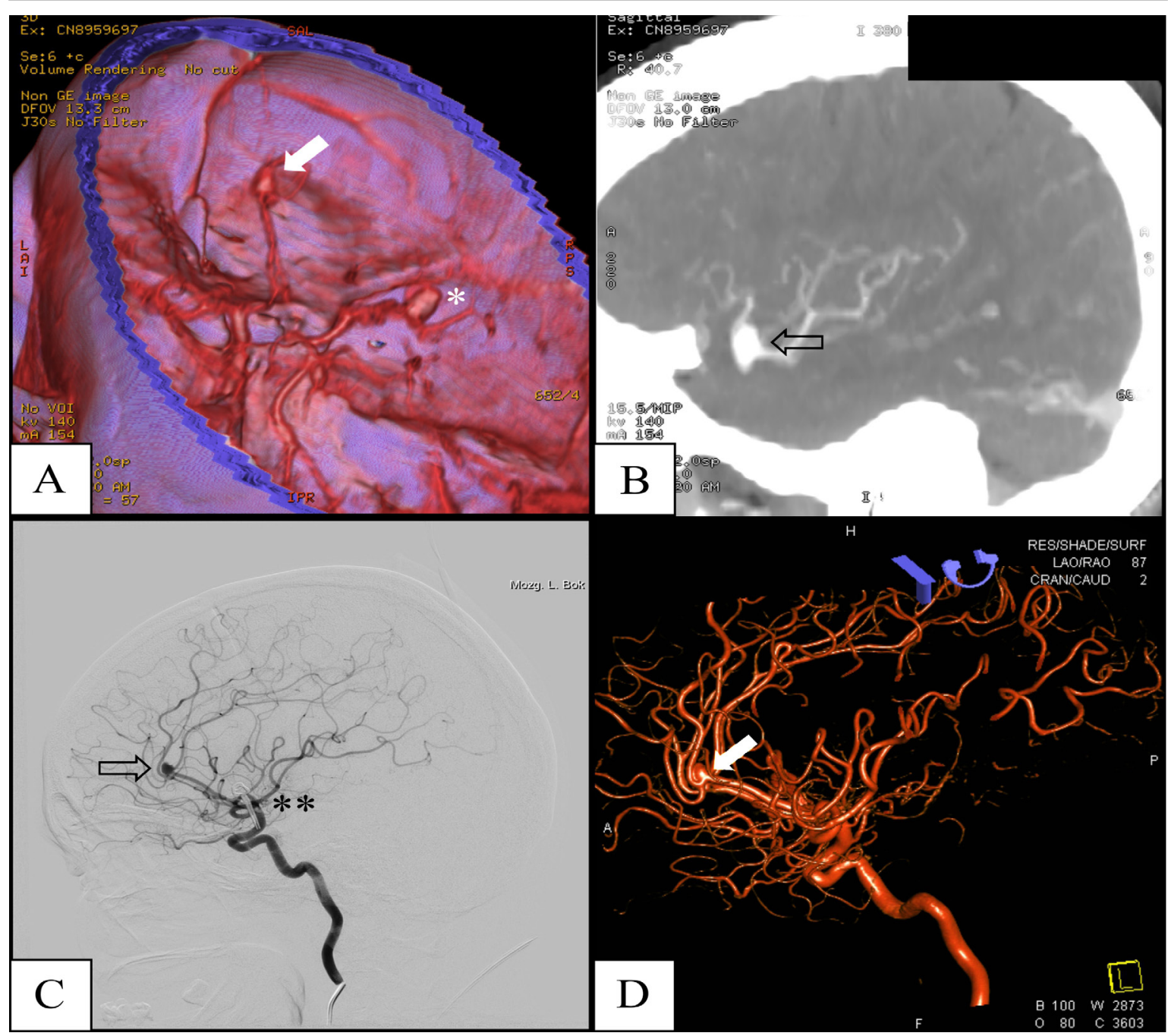

Legend: A a B - Computed tomography angiogram (CTA) from 2012 showing the patient's aneurysms at the division of the middle cerebral artery (MCA) into M1/ $\mathrm{M} 2$ segments $-12 \times 7 \mathrm{~mm}$ (star) and at the bifurcation of the anterior cerebral artery to the pericallosal and callosomarginal arteries (A2/3), so called kissing aneurysms - $4 \times 4.5 \mathrm{~mm}$ (arrow); A - a three-dimensional reconstruction, B - lateral CTA scan showing kissing aneurysms.

C A D - Digital subtraction angiography (DSA) from 2013 showing the same kissing aneurysms (arrow) and a shade of the vascular clip from the previous MCA operation. C - DSA showing the lateral presentation of aneurysms, D - a three-dimensional reconstruction. 
aneurysms were applied without any complications. Vascular patency was confirmed using ICG Pulsion (active ingredient: indocyanine green dye; Pulsion Medical Systems SE); temporary clipping was not applied. No electrophysiological monitoring was used during the surgical clipping. In addition, the surgery was prolonged ( 5 hours).

The early postoperative course was uneventful. Magnetic resonance imaging (MRI) performed on third post-operative day revealed no perfusion disturbances in the area of the surgery.

The patient was discharged from the hospital on the 10th post-operative day in good general condition; neither disability nor neurologic deficit was noticed (o score on the modified Rankin scale). No pre- or post-operative neuropsychological testing was performed. The patient was referred for a follow-up appointment after 10-12 days from the discharge date and a neurosurgical follow-up appointment after 8-10 weeks. The surgical wound healing was complicated by an infection. As a result, the patient received empirical clindamycin treatment. Given the wound infection, a reoperation combined with cranioplasty was performed to evacuate the epidural pus and antibiotic therapy was continued. This post-operative period was uneventful and the patient was scheduled for a subsequent cranial allograft procedure.

\section{Discussion}

Kissing aneurysms derive their name from the specific spatial arrangement of two separate but adjacent malformations. Besides certain congenital predispositions (e.g. Marfan syndrome, Ehlers-Danlos syndome, autosomal dominant polycystic kidney disease etc.), the causes and risk factors of KAs have not been fully defined. ${ }^{8}$ It can be assumed, however, that the risk factors are multifactorial and similar to those of other types of multiple aneurysms. The described patient's gender, history of smoking, hypertension, and an unclear episode of alcohol abuse should all be considered as risk factors for aneurysm formation as well as contribute to the potential for aneurysmal rupture in the future. ${ }^{8,10,11}$

Studies have suggested that $57 \%$ of KAs are not recognized preoperatively. ${ }^{6}$ Digital subtraction angiography (DSA) is the gold standard for detecting small and large aneurysms. In non-emergency situations, DSA is essential to establish whether acute treatment is needed or not, and if so, to select a proper treatment option. Angiograms can, however, be mislea- ding or even negative as described in a similar report.? Computed tomography angiography could also be a helpful tool to visualize such malformations because it provides an option for the non-invasive imaging of KAS'. Importantly, it should be emphasized that CTA can miss aneurysms smaller than $3 \mathrm{~mm}$ or give false positive results.' Recently, more and more unruptured intracranial aneurysms are detected incidentally using MRA but treatment decisions are rarely made based on MRA alone.

Management of unruptured aneurysm remains controversial. ${ }^{3.4}$ Generally, aneurysms smaller than $7 \mathrm{~mm}$ are at a low rupture risk. ${ }^{12}$ However, lesions located in the anterior circulation are at an intermediate risk of rupture. ${ }^{12}$ In this case, taking into consideration the patient's risk factors, the surgical protection of KAs seems desirable. According to Rinkel et al., the relative risks (RR) for aneurysm rupture and their corresponding risk factor in our patient included hypertension (RR 2.0), heavy alcohol intake (RR 2.1), female gender (RR 2.1), smoking (RR 3.4), and the presence of multiple aneurysms (RR 1.7). ${ }^{11}$ The multidisciplinary team decided upon the microsurgical clipping of the aneurysm necks. According to guidelines, 45-year-old patients can benefit from surgical treatment. Endovascular treatment seems to be more challenging in circumstances involving KAs given the two vascular origins without a direct communication and the aneurysms at the distal parts of intracranial vessels (such as $\mathrm{A}_{2} / \mathrm{A}_{3}$ ) being more difficult to treat by coiling.

The preferred treatment method for a single unruptured aneurysm remains controversial; thus, an unruptured KA makes the decision process even more complicated. ${ }^{3}$ Endovascular treatment has lower overall unfavorable outcomes but the patient's age seems to be a crucial factor. ${ }^{13}$ Surgery-related morbidity and mortality are quite low among patients under 60 years of age. ${ }^{13}$ However, endovascular procedures can lead to an increased risk of recurrence or retreatment in this patient group. ${ }^{13,14}$ It is noteworthy that the surgical procedure provides the neurosurgeon with access and space for more maneuvers within the surroundings of the bilateral lesion. Surgery is difficult due to the dual arterial supply of KAs. ${ }^{5}$ The procedure should secure both aneurysms simultaneously; however, this maneuver can lead to a higher risk of intraoperative bleeding. ${ }^{5}$

The patient had regular (every 6 months) follow-up care at the Neurosurgery Outpatient Clinic. Computed tomography angiography examination was performed after 12 months, showing complete aneurysm occlusion with no new pathologies. Althou-

Figure 2. Surgical Clipping of Aneurysms - Intraoperative View.

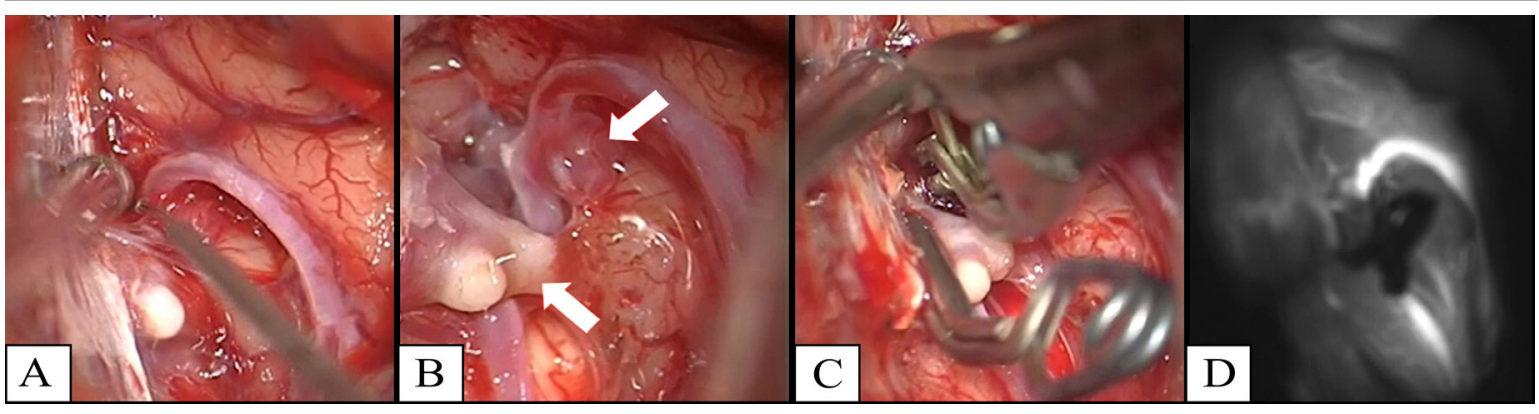

Legend: A - The pia mater was carefully dissected and the pericallosal artery was exposed.

$B$ - Both aneurysms at the bifurcation of the anterior cerebral artery into the pericallosal and callosomarginal arteries are visible (arrows).

$B$ - Both aneurysms at the bifurcation of the anterior cer
C - Subsequently, their necks have both been clipped.

C - Subsequently, their necks have both been clipped.
D - Vascular patency was confirmed using ICG Pulsion (active ingredient: indocyanine green dye). 


\section{Case Report}

gh the re-treatment ratio (short-term prognosis) after the successful surgical clipping is low, the patient is at risk of aneurysm recurrence (long-term prognosis). ${ }^{14}$ So far (after 1.5 years of observation), the patient's follow-up remains uneventful.

Kissing aneurysms are a treatment challenge because of their unknown etiology, difficult imaging diagnosis, and no officially stated treatment method. In our opinion, every case has to be considered individually by an interdisciplinary medical team. In order to assure positive outcomes, patients with this malformation should be referred to an experienced neurosurgical center. Further studies are needed to allow for a better understanding of this condition and the therapeutic options available to patients with KAs. 


\section{References}

1. Vlak MH, Algra A, Brandenburg R, Rinkel GJ. Prevalence of unruptured intracranial aneurysms, with emphasis on sex, age, comorbidity, country, and time period: A systematic review and meta-analysis. Lancet Neurol. 2011 Jul;10(7):626-36.

2. Koffijberg H, Buskens E, Granath F, Adami J, Ekbom a, Rinkel G], et al. Subarachnoid haemorrhage in Sweden 1987-2002: regional incidence and case fatality rates. J Neurol Neurosurg Psychiatry. 2008 Mar;79(3):294-9.

3. Brown RD, Broderick JP. Unruptured intracranial aneurysms: epidemiology, natural history, management options, and familial screening. Lancet Neurol. 2014 Apr;13(4):393-404.

4. Darsaut TE, Estrade L, Jamali S, Bojanowski MW, Chagnon M, Raymond J. Uncertainty and agreement in the management of unruptured intracranial aneurysms. J Neurosurg. 2014 Mar;120(3):618-23.

5. Baldawa SS, Menon G, Nair S. Kissing anterior communicating artery aneurysms: diagnostic dilemma and management issues. J Postgrad Med. 2011 Jan-Mar;57(1):44-7.

6. Harada K, Orita T, Ueda Y. [Large kissing aneurysms of the middle cerebral artery: a case report--classification of kissing aneurysms]. No Shinkei Geka. 2004 May;32(5):513-7. Jap

7. Alimohammadi M, Bidabadi MS, Amirjamshidi A. Bilateral "Kissing" Aneurysms of the Distal Pericallosal Arteries: Report of a Case and Review of the Literature. Neurosurg Q. 2010;20(4):308-10.
8. Casimiro MV, McEvoy AW, Watkins LD, Kitchen ND. A comparison of risk factors in the etiology of mirror and nonmirror multiple intracranial aneurysms. Surg Neurol. 2004 Jun;61(6):541-5.

9. Salunke P, Malik V, Yogesh N, Khandelwal NK, Mathuriya SN. Mirror-like aneurysms of proximal anterior cerebral artery: report of a case and review of literature. Br J Neurosurg. 2010 Dec;24(6):686-7.

10. Vlak MHM, Rinkel GJ, Greebe P, Greving JP, Algra A. Lifetime risks for aneurysmal subarachnoid haemorrhage: multivariable risk stratification. J Neurol Neurosurg Psychiatry. 2013 Jun;84(6):619-23.

11. Rinkel GJ, Djibuti M, Algra A, van Gijn J. Prevalence and risk of rupture of intracranial aneurysms: a systematic review. Stroke. 1998 Jan;29(1):251-6. 12. Bijlenga $P$, Ebeling $C$, Jaegersberg $M$, Summers $P$, Rogers $A$, Waterworth $A$, et al. Risk of rupture of small anterior communicating artery aneurysms is similar to posterior circulation aneurysms. Stroke. 2013 Nov;44(11):3018-26. 13. Lawson MF, Neal DW, Mocco J, Hoh BL. Rationale for treating unruptured intracranial aneurysms: Actuarial analysis of natural history risk versus treatment risk for coiling or clipping based on 14,050 patients in the nationwide inpatient sample database. World Neurosurg. 2013 Mar-Apr;79(3-4):472-8. 14. Corns R, Zebian B, Tait MJ, Walsh D, Hampton T, Deasy N, et al. Prevalence of recurrence and retreatment of ruptured intracranial aneurysms treated with endovascular coil occlusion. Br J Neurosurg. 2013 Feb;27(1):30-3.

\section{Acknowledgments}

None.

Conflict of Interest Statement $\mathrm{C}$ Funding

The author has no funding, financial relationships or conflicts of interest to disclose.

\section{Author Contributions}

Conception and design the work/idea: PMW, AP, KA. Collect data/obtaining results, Analysis and interpretation of data: PMW, AP, KB, BB. Write the manuscript: PMW, AP, JS, KB, BB. Critical revision of the manuscript: PMW, JS, KA. Approval of the final version: PMW, JS. Administrative or technical advice: JS.

Cite as:

Waszak PM, Paturej A, Springer J, Baranowska K, Brzeska B, Aleksandrowicz K, Szmuda T, Garnier H. Romantic Name for a Deadly Condition: Kissing Aneurysms of the Pericallosal Artery - A Case Report. Int J Med Students. 2015 Apr-Aug;3(2):107-11. 